\title{
How long do multirooted teeth with furcation involvement survive with treatment?
}

\author{
Abstracted from \\ Huynh-Ba G, Kuonen P, Hofer D, Schmid J, Lang NP, Salvi GE. \\ The effect of periodontal therapy on the survival rate and incidence of complications of \\ multirooted teeth with furcation involvement after an observation period of at least 5 years: \\ a systematic review. J Clin Periodontol 2009; 36: 164-176 \\ Address for correspondence: Giovanni E Salvi, Department of Periodontology, \\ School of Dental Medicine, University of Bern, Freiburgstrasse 7, CH-3010 Bern, \\ Switzerland. E-mail: giovanni.salvi@zmk.unibe.ch
}

\section{Question: In people undergoing periodontal treatment of multirooted teeth with furcation involvement, what is the survival rate and incidence of complication at 5 years?}

Data sources A Medline search and handsearching of the following journals were carried out: International Journal of Periodontics and Restorative Dentistry, Journal of Clinical Periodontology, Journal of Periodontal Research and Journal of Periodontology as well as reference lists of publications selected.

Study selection To be eligible for inclusion in this review, studies had to be longitudinal in nature. Prospective and retrospective cohort studies were considered. Studies were screened and quality assessed independently by two reviewers. Review articles, case reports and studies of fewer than 5-years' duration were excluded, as were those not providing information on tooth survival or furcation involvement. Data extraction and synthesis Data was abstracted independently by two reviewers. Owing to the heterogeneity of the data, a metaanalysis could not be performed. A qualitative synthesis was conducted grouping the studies into the following areas: nonsurgical furcation therapy; surgical therapy not involving tooth structures; tunnelling surgical resective therapy (eg, root resection and/ or root separation); and guided tissue regeneration (GTR) and grafting procedures. Results Twenty-two publications met the inclusion criteria. The survival rate of molars treated nonsurgically was $>90 \%$ after 5-9 years. The corresponding values for the different surgical procedures were: surgical therapy, 43.1-96\% (observation period, 5-53 years); tunnelling procedures, 42.9-92.9\% (observation period, 5-8 years); surgical resective procedures including amputation(s) and hemisections, $62-100 \%$ (observation period, 5-13 years); and GTR, $83.3-100 \%$ (observation period, $5-12$ years). The most frequent complications included caries in the furcation area after tunnelling procedures and root fractures after root-resective procedures. Conclusions Good long-term survival rates (up to 100\%) of multirooted teeth with furcation involvement were obtained following various therapeutic approaches. Initial furcation involvement (degree I) could be successfully managed by nonsurgical mechanical debridement. Vertical root fractures and endodontic failures were the most frequent complications observed following resective procedures.

\section{Commentary}

This investigation is interesting because it attempts to provide data that could be helpful to clinical decision-making. Furcationinvolved teeth are harder to treat than nonfurcation-involved ones. Should we offer such treatment to patients and, if so, what can be expected? Furthermore, with tooth replacement options such as dental implants available, how can informed choices be made?

So, how good is the systematic review? First, it is important to note the difficulty of reviewing this topic, resulting from a number of factors: there are no trials, and the available studies are mostly retrospective (with much greater potential for bias) and conducted over a 35-year span. Also, there are substantial differences between the included studies, which limits the potential to generalise conclusions. Despite the problems with the literature, however, the authors were correct to carry out a systematic review in an attempt to produce 'best evidence'.

The strengths of the review are that it brings together a well-established team with appropriate skills for this research. They decided not to conduct meta-analysis on the basis of the heterogeneity of the studies (as well as their potential for bias) and this seems sensible. Carrying out a meta-analysis is possible but would most likely produce both a false sense of precision and a questionable estimate of how well treatments worked.

The limitations of the review are potentially important and require good judgement when interpreting the possible impact of the authors' conclusions. The main issues are the electronic search and its risk of bias/ quality assessment. The search was limited to one electronic database, Medline. It should be routine to search other databases for systematic reviews, in particular Embase (around 40\% of journals indexed in Embase are not found in Medline) and CENTRAL, the Cochrane Collaboration database. The risk with searching Medline alone is of missing important data, with resulting greater uncertainty accompanying the review's conclusions. The authors did handsearch four main journals of periodontology from 1975-2008, as well as checking the reference lists of retrieved papers, which is helpful, although unlikely to identify papers published in journals not indexed in Medline. My impression is that they found most of the relevant publications and, in fact, additional studies would not have changed the conclusions.

Quality assessment of included studies is a fundamental property of a systematic review and one that distinguishes it from a traditional review. As a reader, the question that I would like an answer to is, can I trust the findings of the individual 
studies? For this review, the domains that I would be interested in are risk of bias, use of appropriate statistical methods, were the patients selected (sample) the right ones and was the duration of followup sufficient to detect the outcome (tooth retention)? The authors of this review do not present such an assessment and don't really take the opportunity to interpret the results for us in the light of the quality of the scientific literature. Even without reading the original studies, however, the fact that they are mostly retrospective carries a high risk of bias, which is likely to be in the direction of overly optimistic findings. We cannot comment on appropriateness of statistical methods. The lack of details regarding the types of patients limits our knowledge of the generalisability of the conclusions, although studies represent both university and private specialist clinics. The duration of followup was good: 5 years would seem a sensible minimum period.
Overall, then, this review is valuable both in identifying the literature on the effect of periodontal treatment on retention of furcation involved teeth. The data suggest high levels of tooth retention comparable with implant-supported restorations in the posterior mandible and maxilla. The level of evidence is not high and there may be an overestimation of true retention rates, but similar comments are applicable to much of the relevant implant literature.

Ian Needleman

International Centre for Evidence-Based Oral Health and Unit of Periodontology, Division of Restorative Dental Sciences, University College London Eastman Dental Institute, London, UK

Evidence-Based Dentistry (2010) 11, 38-39. doi:10.1038/sj.ebd.6400714 